\title{
Dehydroepiandrosterone (DHEA) reduces embryo aneuploidy: direct evidence from preimplantation genetic screening (PGS)
}

Norbert Gleicher ${ }^{1,2^{*}}$, Andrea Weghofer ${ }^{1,3}$, David H Barad ${ }^{1,4}$

\begin{abstract}
Background: Dehydroepiandrosterone (DHEA) has been reported to improve pregnancy chances in women with diminished ovarian reserve (DOR), and to reduce miscarriage rates by $50-80 \%$. Such an effect is mathematically inconceivable without beneficial effects on embryo ploidy. This study, therefore, assesses effects of DHEA on embryo aneuploidy.

Methods: In a 1:2, matched case control study 22 consecutive women with DOR, supplemented with DHEA, underwent preimplantation genetic screening (PGS) of embryos during in vitro fertilization (IVF) cycles. Each was matched by patient age and time period of IVF with two control IVF cycles without DHEA supplementation ( $n=$ 44). PGS was performed for chromosomes $X, Y, 13,16,18,21$ and 22, and involved determination of numbers and percentages of aneuploid embryos.
\end{abstract}

Results: DHEA supplementation to a significant degree reduced number $(P=0.029)$ and percentages $(P<0.001)$ of aneuploid embryos, adjusted for relevant covariates. Short term supplementation (4-12 weeks) resulted in greatest reduction in aneuploidy $(21.6 \%, 95 \% \mathrm{Cl}-2.871-46.031)$.

Discussion: Beneficial DHEA effects on DOR patients, at least partially, are the likely consequence of lower embryo aneuploidy. DHEA supplementation also deserves investigation in older fertile women, attempting to conceive, where a similar effect, potentially, could positively affect public health.

\section{Background}

Dehydroepiandrosterone (DHEA) has been demonstrated to improve embryo quality and pregnancy chances in women with diminished ovarian reserve (DOR) [1-3]. How these effects are achieved is, however, unknown. A small pilot study of limited power suggested that DHEA may reduce aneuploidy [4]. Since aneuploidy in human embryos is frequent and increases with advancing female age [5,6], a reduction in aneuploidy could, at least partially, explain improved embryo quality and pregnancy rates.

Aneuploidy in preimplantation embryos can be demonstrated through preimplantation genetic screening (PGS) [7]. PGS is, however, only rarely indicated in women with DOR, where often only small embryo

\footnotetext{
* Correspondence: ngleicher@thechr.com

${ }^{1}$ Center for Human Reproduction (CHR) - New York and the Foundation for Reproductive Medicine, New York, NY, USA

Full list of author information is available at the end of the article
}

numbers are available, and embryo selection, therefore, does not offer clinical benefits [8]. PGS in such cases may, actually, reduce pregnancy chances with in vitro fertilization (IVF) [9].

Our initial pilot study, attempting to investigate DHEA effects on ploidy, was underpowered. In that study we were able to demonstrate that DHEA supplemented patients had greater chances of at least one euploid embryo. The study, likely because of small patient numbers, however, failed to demonstrate significant decreases in overall aneuploidy [4].

For lack of adequate PGS case numbers, we, therefore, pursued an alternative strategy by investigating miscarriage rates after DHEA supplementation as a surrogate for aneuploidy risk [10]. Since at least 60 percent of spontaneous pregnancy loss is attributable to chromosomal abnormalities [11], we hypothesized that significant reductions in aneuploidy after DHEA supplementation should be reflected in lower miscarriage rates. This was, 
indeed, confirmed in a study, involving patients from two independent centers in New York City and Toronto, Canada [10].

While results of this study were strongly supportive of a DHEA effect on aneuploidy, they were unable to offer direct evidence, which can only come from PGS studies of human embryos. Such a study is presented here.

\section{Methods}

\section{Patient populations}

We retrieved from our center's computerized research data bank a total of 22 consecutive DOR patients who underwent IVF/PGS while on DHEA supplementation. Only first IVF cycles were analyzed. These cycles were matched with the two control cycles not on DHEA supplementation, based on patient age and year of treatment (44 controls). Primary medical records for all of these patients were pulled and manually reviewed by one of the authors (A.W.).

A diagnosis of DOR was reached if patients demonstrated abnormally elevated age-specific baseline follicle stimulating hormone (FSH) or abnormally low age-specific anti-Müllerian hormone (AMH) levels. Normal age-specific hormone levels were defined by $95 \%$ confidence intervals at all ages, as previously reported $[12,13]$. Since patients with DOR were, thus, uniformly diagnosed before IVF cycle protocols were determined, they were all supplemented with DHEA, and stimulation adjustments were made. As we previously reported, this results in improved oocyte and embryo yields in comparison to patients who are not diagnosed with use of age-specific FSH and AMH $[12,13]$.

\section{DHEA supplementation}

During the study period all DOR patients at our center routinely received DHEA supplementation [14]. Those not receiving DHEA, therefore, by definition, had ageappropriate ovarian reserve, confirmed by normal antiMüllerian hormone (AMH), follicle stimulating hormone (FSH) baseline levels and estradiol. Patients receiving DHEA supplementation were prescribed 25 mg of micronized, pharmaceutical grade DHEA, T.I.D, for at least four weeks prior to IVF cycle start [14]. Short-term supplementation was defined as 4 to 12 weeks of DHEA prior to IVF and PGS; supplementation beyond that was considered long-term supplementation.

\section{Ovarian stimulation}

Patients with premature DOR, or if over 40 years of age, in first treatment cycles universally receive a so-called microdose gonadotropin releasing hormone agonist (GnRH-a) ovarian stimulation protocol, characterized by leuprolide acetat $\left(50 \mu \mathrm{g} / 0.1 \mathrm{~mL}\right.$, b.i.d.; Lupron ${ }^{\circ}$, Abbot Pharmaceuticals, North Chicago, IL) and ovarian stimulation with follicle stimulating hormone (FSH, 300 IU-450 IU daily) and human menopausal gonadotropins (hMG, $150 \mathrm{IU}$ ). If under age 40 with normal ovarian reserve patients receive down regulation with full dose GnRH-a $(1.0 \mathrm{mg} / 0.1 \mathrm{~mL})$ and ovarian stimulation with up to $300 \mathrm{IU}$ of gonadotropins, usually half as FSH and half as hMG. Higher luteinizing hormone (LH) contributions to ovarian stimulation, if anything, reduce embryo aneuploidy [15]. The small difference in ovarian stimulation protocols between women with DOR and controls, therefore, potentially biases study outcome towards lower aneuploidy rates in the control population, which received a proportionally higher LH contributions to ovarian stimulation.

\section{Preimplantation genetic screening (PGS)}

PGS was performed, utilizing fluorescence in situ hybridization (FISH) in routine fashion, utilizing probes for seven chromosomes (X, Y, 13, 16, 18, 21 and 22) on day three after fertilization, when embryos reached six to eight cell stages. This restricted chromosome panel is currently routinely utilized for PGS [15].

\section{Statistical analysis}

A general linear model was constructed to assess DHEA effects on percent aneuploidy after adjustment for age, indications for PGS, stimulation protocol and total gonadotropin dosage utilized. The latter adjustment was made as a surrogate for potential physician biases in how individual patients were stimulated, and potential effects such stimulation biases may have on ploidy $[15,16]$.

All patients at our center sign a universal informed consent at time of initial presentation, which permits the extraction of clinical data from patient records as long as confidentiality of the record and anonymity of patients is maintained. The center's Institutional Review Board, therefore, permits such studies under expedited review.

\section{Results}

\section{Patients}

Table 1 summarizes characteristics of study and control patients. The two groups did not differ in age and race/ ethnicity. DHEA patients were significantly more obese but expressed poorer ovarian reserve, based on lower AMH $(\mathrm{P}=0.045)$ and significantly higher gonadotropin utilization $(P=0.002)$. Such a conclusion was also supported by trends towards higher FSH and smaller oocyte yields $(9.6 \pm 6.2$ vs. $11.7 \pm 6.3)$. Embryo numbers transferred ( $1.4 \pm 0.9$ vs. $1.5 \pm 0.7$ ), embryos cryopreserved ( $0.7 \pm 1.6$ vs. $0.6 \pm 1.2)$, embryos undergoing PGS $(7.3 \pm$ 3.9 vs. $6.6 \pm 3.6)$ and embryo grades $(3.4 \pm 0.4$ vs. $3.5 \pm$ 0.3 ) were similar between both groups. 
Table 1 Patient characteristics

\begin{tabular}{|c|c|c|c|}
\hline & DHEA & Controls & $\begin{array}{c}\mathrm{P}- \\
\text { value }\end{array}$ \\
\hline Number & 22 & 44 & \\
\hline Age (years, Mean \pm SD) & $37.9 \pm 4.7$ & $37.2 \pm 4.4$ & N.S. \\
\hline \multicolumn{4}{|l|}{ Race/ethnicity (\%) } \\
\hline Caucasian & $14(63.6)$ & $21(47.7)$ & \\
\hline African & $2(9.1)$ & $3(6.8)$ & \\
\hline Asian & $4(18.2)$ & $14(31.8)$ & \\
\hline Middle Eastern & $1(4.5)$ & $6(13.6)$ & \\
\hline BMI (Mean \pm SD) & $24.4 \pm 3.8$ & $21.0 \pm 1.7$ & 0.006 \\
\hline $\mathrm{AMH}(\mathrm{ng} / \mathrm{mL}$, mean $\pm \mathrm{SD})$ & $1.3 \pm 1.2$ & $2.0 \pm 1.9$ & 0.045 \\
\hline Range & $0.8-2.1$ & $1.0-4.4$ & \\
\hline $\mathrm{FSH}(\mathrm{mlU} / \mathrm{mL}$, mean $\pm \mathrm{SD})$ & $10.1 \pm 6.8$ & $8.0 \pm 5.5$ & N.S. \\
\hline Range & $8.4-12.2$ & $7.1-9.1$ & \\
\hline Oocytes retrieved ( $n$, mean $\pm S D$ ) & $9.6 \pm 6.2$ & $11.7 \pm 6.3$ & N.S. \\
\hline \multicolumn{4}{|l|}{ Embryos $(\mathrm{n}$, mean $\pm \mathrm{SD})$} \\
\hline Transferred & $1.4 \pm 0.9$ & $1.5 \pm 0.7$ & \\
\hline Cryopreserved & $0.7 \pm 1.6$ & $0.6 \pm 1.2$ & \\
\hline Undergoing PGS & $7.3 \pm 3.9$ & $6.6 \pm 3.6$ & \\
\hline Grades & $3.4 \pm 0.4$ & $3.5 \pm 0.3$ & \\
\hline $\begin{array}{l}\text { Total gonadotropins } \\
\text { dosage (IU, mean } \pm \text { SD) }\end{array}$ & $\begin{array}{c}5711 \pm \\
1818\end{array}$ & $\begin{array}{c}4048 \pm \\
1886\end{array}$ & 0.002 \\
\hline
\end{tabular}

${ }^{1}$ Difference in BMI was based on significant difference in weight $(P=0.02)$ not height $(P=0.24)$

\section{Aneuploidy}

As Figure 1, however, demonstrates, aneuploid embryos were significantly more prevalent amongst controls (4.5 \pm 3.1 vs. $2.8 \pm 2.5 ; \mathrm{P}=0.03)$, as were percentages of aneuploidy $(61.0 \pm 22.4$ vs. $38.2 \pm 24.4 ; \mathrm{P}<0.001)$. In the general linear model, after adjustment for age, FSH dose and indication for PGS, the association of DHEA supplementation effects on ploidy remained significant $(\mathrm{F}=13.2$, df $1, \mathrm{p}=0.001)$. As expected, women who underwent PGS for aneuploidy screening had a greater percentage of aneuploidy embryos than women who underwent PGS for elective gender selection purposes $(\mathrm{P}<0.007)$.

Possibly because of still relatively small study numbers, no specific aneuploidy pattern, affecting distinct chromosomes, was apparent.

Mean length of DHEA supplementation was $7.3 \pm 2.2$ weeks in the short and $19.1 \pm 9.1$ weeks in the long treatment group. Women in the short treatment group demonstrated the greatest reduction in aneuploidy (21.6\%, 95\%CI -2.871-46.031).

\section{Discussion}

This study supports prior preliminary evidence that DHEA supplementation reduces aneuploidy in women with DOR, first suggested in a small pilot study, when at least one euploid embryo was found significantly more frequently after DHEA than in matched control

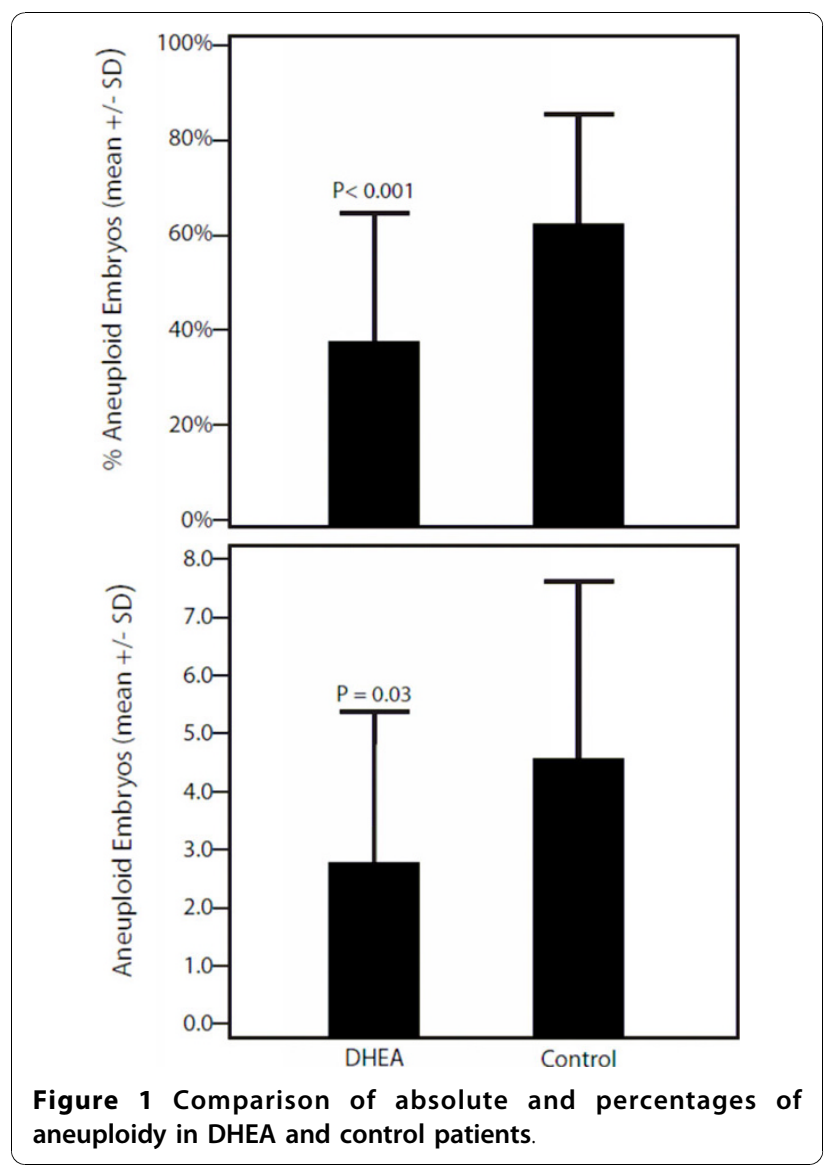

cycles [4]. Subsequently, we also demonstrated that DHEA supplementation reduces miscarriage rates to a degree that cannot be explained without significant contribution from reduced aneuploidy [10].

By demonstrating no difference in embryo grades between DHEA and control cycles (Table 1), this study also demonstrates, once more, that embryo morphology, as currently routinely assessed in most IVF laboratories, does not reflect on embryo ploidy and, therefore, is limited in clinical value.

Since a majority of miscarriages are believed to be consequence of aneuploidy [11], decreases in aneuploidy rate should translate into decreases in spontaneous pregnancy loss. Two infertility centers utilizing DHEA supplementation, one in New York City and the other in Toronto, Canada, indeed, independently, reported identically low miscarriage rates of 15.0 and 15.2 percent, respectively. Depending on method of statistical analysis, these miscarriage rates represented declines of approximately 50 to 80 percent from expectations [10]. Even more remarkably, the combined loss rate of 15.1 percent equated rates reported for normal populations as young as 28 to 33 years [17], and was, thus, far 
removed from excessively high miscarriage rates, reported in DOR patients [18].

Even though such significant declines in spontaneous miscarriages cannot be achieved without underlying improvements in aneuploidy, miscarriage rates only represent surrogates for true aneuploidy studies. Direct evidence for such an effect was, therefore, still needed.

In this study we for the first time are able to demonstrate such direct evidence, utilizing routinely performed PGS of preimplantation stage embryos, performed in DHEA supplemented IVF cycles and controls. DHEA supplementation was, indeed, associated with significantly reduced aneuploidy, and greatest reductions were observed with short DHEA supplementation of up to 12 weeks.

This observation on first impulse suggests that, excluding month one of supplementation, second and third months offer the best chance of lowering aneuploidy, thus fully supporting previously published pregnancy data after DHEA supplementation, which demonstrated a significant first rise in pregnancy rate after approximately six weeks of DHEA supplementation [14]. Six weeks of DHEA supplementation prior to IVF cycle start, therefore, currently represents minimal supplementation time at our center.

This study, however, does not preclude, as alternative explanation for these findings that a more favorable patient group conceives quickly and, therefore, statistically distorts above noted time associations. Such a possibility cannot be ruled out since we previously demonstrated that women who improve AMH levels with DHEA supplementation demonstrate significantly superior pregnancy rates to those who do not [19].

A general criticism of currently available technologies for PGS is that only limited numbers of chromosomes can be evaluated (24 chromosome screening technologies are currently under investigation). In this study this meant that only seven chromosomes were assessed in study and control patients. This allows for the at least theoretical possibility that untested chromosomes demonstrate statistically different aneuploidy distributions from the here tested seven and that, including a full chromosome complement, here reported differences would disappear. Such an explanation is, however, highly unlikely, and the here utilized selection of chromosomes, or similar ones, have been routinely used in clinical PGS $[15,16]$. There is also no data in either human or animal literature to suggest that DOR maybe associated with aneuploidy of specific chromosomes.

Because of time pressures, when using their own oocytes, prospectively randomized clinical trials in patient populations affected by DOR, and involving placebo, are difficult, if not impossible, to conduct. Our center, for that reason, had to abandon two registered clinical DHEA trials, one in the United States and one in Europe, due to lack of enrollment [14]. A small first such trial has just been reported [3]. Best available evidence, therefore, at least in part, has to be obtained via other study formats.

In this study, the format chosen was a case control study in which each study patient/cycle was matched with two controls. As Table 1 demonstrates, patient and control populations appear, with few exceptions, overall comparable. It is, however, important to point out that the significantly larger preponderance of DOR in the study group (Table 1) biases study results against discovery of DHEA effects on ploidy since DOR patients demonstrate the highest aneuploidy rate amongst infertility patients [18]. Even just absence of increased aneuploidy in the study group could, therefore, be viewed as a potentially positive DHEA effect. Instead, this study actually demonstrates significantly lower aneuploidy following DHEA supplementation.

How DHEA affects non-dysfunctional events remains to be determined but we have speculated that DHEA supplementation may improve the ovarian environment in which follicular maturation takes place in older women [19 and submitted]. DHEA, indeed, significantly declines with advancing age [20]. Since DHEA, except in our prior pilot study [4], has never before been directly associated with decreases in aneuploidy, neither animal nor human data are currently available to speculate further on specific mechanisms that may be involved.

Others have speculated that drugs can be developed which beneficially affect non-dysjunctional events during meiosis [21]. DHEA may, indeed, turn out to be a first pharmacologic agent to do so.

This effect, only unlikely, should be restricted to infertile women with DOR. DHEA supplementation, in attempts to reduce embryo aneuploidy and spontaneous miscarriages, therefore, also deserves investigation in, especially older (above age 35 years) fertile women, attempting conception. A possible similar beneficial impact in fertile patient populations, attempting spontaneous conception, could have a major impact on public health by speeding up time to pregnancy and by reducing embryo aneuploidy and miscarriage rates.

\section{Abbreviations}

AMH: anti-Müllerian hormone; DHEA: dehydroepiandrosterone; DOR: diminished ovarian reserve; FISH: fluorescence in situ hybridization; FSH: follicle stimulating hormone; GnRH-a: gonadotropins releasing hormone agonist; hMG: human menopausal gonadotropins; I.U.: international units; IVF: in vitro fertilization; $\mathrm{mL}$ : milliliter; T.I.D.: three-times daily

\section{Acknowledgements}

This study was supported by the Foundation for Reproductive Medicine and intramural research funds from the Center for Human Reproduction, New York. 


\section{Author details}

${ }^{1}$ Center for Human Reproduction (CHR) - New York and the Foundation for Reproductive Medicine, New York, NY, USA. ${ }^{2}$ Department of Obstetrics, Gynecology and Reproductive Sciences, Yale University School of Medicine, New Haven, CT, USA. ${ }^{3}$ Department of Obstetrics and Gynecology, Vienna University School of Medicine, Vienna, Austria. ${ }^{4}$ Departments of Epidemiology and Social Medicine and Obstetrics, Gynecology and Women's Health, Albert Einstein College of Medicine, Bronx, NY, USA.

\section{Authors' contributions}

NG, AW and DHB contributed equally to the manuscript. NG, AW, DHB conceived and designed the study. AW conducted chart reviews. DHB performed statistical analyses. NG wrote manuscript. All three authors contributed, reviewed and approved the manuscript. All authors read and approved the final manuscript.

\section{Competing interests}

NG and DHB are listed as co-inventors on a number of U.S. patent applications (one already awarded), claiming therapeutic benefits from DHEA supplementation in women with diminished ovarian reserve (DOR). NG is owner of $\mathrm{CHR}$, where this research was conducted. NG, DHB and AW received research grants, travel reimbursements and speaker honoraria from various pharmaceutical companies, none, however, related to DHEA or any other issues addressed in this manuscript. None of the authors has any formal links with pharmaceutical companies and/or owns shares in pharmaceutical companies.

Received: 1 October 2010 Accepted: 10 November 2010 Published: 10 November 2010

\section{References}

1. Barad D, Gleicher N: Effect of dehydroepiandrosterone on oocytes and embryo yields, embryo grade and cell number in IVF. Hum Reprod 2006, 21:2845-2849.

2. Barad D, Brill H, Gleicher N: Update on the use of dehydroepiandrosterone supplementation among women with diminished ovarian function. J Assist Reprod Genet 2007, 24:629-634.

3. Wiser A, Gonen O, Ghetler Y, Shavit T, Berkovitz A, Shulman A: Addition of dehydroepiandrosterone (DHEA for poor-responder patients before and during IVF treatment improves the pregnancy rate: a randomized prospective study. Hum Reprod 2010, 25:2496-2500.

4. Gleicher N, Weghofer A, Barad D: Increased euploid embryos after supplementation with dehydroepiandrosterone (DHEA) in women with premature ovarian aging. Fertil Steril 2007, 88(Suppl 1):S232.

5. Eichenlaub-Ritter U: Parentral age-related aneuploidy in human germ cells and offspring: a story of past and present. Environ Mol Mutagen 1996, 28:211-236.

6. Wyrobek AJ, Aardema M, Eichenlaub-Ritter U, Ferguson L, Marchetti F: Mechanisms and targets involved in maternal and paternal age effects on numerical aneuploidy. Environ Mol Mutagen 1996, 28:254-264.

7. Twisk M, Mastenbroek S, van Wely M, Heineman MJ, Van der Veen F, Repping S: Preimplantation genetic screening for abnormal number of chromosomes (aneuploidies) in in vitro fertilization or intracytoplasmic sperm injection. Cochrane Database Syst Rev 2006, 25(1):CD005291.

8. Gleicher N, Weghofer A, Barad D: Preimplantation genetic screening: "established" and ready for prime time? Fertil Steril 2008, 89:780-788.

9. Mastenbroek S, Twisk M, van Echten-Arends J, Sikkema-Raddatz B, Korevaar JC, Verhoeven HR, Vogel NE, Arts EG, de Vries JW, Bossuyt PM, Buys $C H$, Heineman MJ, Repping $S$, van der Veen F: In vitro fertilization with preimplantation genetic screening. N Engl J Med 2007, 357:9-17.

10. Gleicher N, Ryan E, Weghofer A, Blanco-Mejia S, Barad DH: Miscarriage rates after dehydroepiandrosterone (DHEA) supplementation in women with diminished ovarian reserve: a case control study. Reprod Biol Endocrinol 2009, 7:108.

11. Bettio D, Venci A, Levi Setti PE: Chromosomal abnormalities in miscarriages after different assisted reproduction procedures. Placenta 2008, 29(Suppl B):126-8.

12. Barad DH, Weghofer A, Gleicher N: Age-specific levels for basal folliclestimulatng hormone assessment of ovarian function. Obstet Gynecol 2007, 109:14014-1410
13. Barad DH, Weghofer A, Goyal A, Gleicher N: Age-specific anti-Müllerian hormone (AMH): Utility of AMH at various ages. Reprod Biomed Online.

14. Barad D, Brill H, Gleicher N: Update on the use of dehydroepiandrosterone supplementation among women with diminished ovarian function. J Assist Reprod Genet 2007, 24:629-634.

15. Weghofer A, Munné S, Brannath W, Chen S, Tomkin G, Cekleniak N, Garrisi M, Barad D, Cohen J, Gleicher N: The impact of LH-containing gonadotropins on diploidy rates in preimplantation embryos: long protocol stimulation. Hum Reprod 2008, 23:499-503.

16. Weghofer A, Munné S, Brannath W, Chen S, Barad D, Cohen J, Gleicher N: The impact of LH-containing gonadotropins stimulation on euploidy rates in preimplantation embryos: antagonist cycles. Fertil Steril 2009, 92:937-942.

17. Herbert D, Lucke J, Dobson A: Pregnancy losses in young Australian women: findings from the Australian Longitudinal Study of Women's Health. Womens Health Issues 2009, 19:21-29.

18. Levi AJ, Raynault MF, Bergh PA, Drews MR, Miller BT, Scott RT Jr: Reproductive outcome in patients with diminished ovarian reserve. Fertil Steril 2001, 76:666-669.

19. Gleicher N, Weghofer A, Barad DH: Improvement in diminished ovarian reserve after dehydroepiandrosterone (DHEA) supplementation. Reprod Biomed Online 2010, 21:360-365.

20. Jacob MH, da R, Janner D, Jahn MP, Kucharski LC, Belló-Klein A, Ribeiro MF: Age-related effects of DHEA on peripheral markers of oxidative stress. Cell Biochem Funct 2010, 28:52-57.

21. Hodges CA, llagan A, Jenninger D, Keri R, Nilson J, Hunt PA: Experimental evidence that changes in oocyte growth influence meiotic chromosome segregation. Hum Reprod 2002, 17:1171-1180.

doi:10.1186/1477-7827-8-140

Cite this article as: Gleicher et al: Dehydroepiandrosterone (DHEA) reduces embryo aneuploidy: direct evidence from preimplantation genetic screening (PGS). Reproductive Biology and Endocrinology 2010 8:140.

\section{Submit your next manuscript to BioMed Central and take full advantage of:}

- Convenient online submission

- Thorough peer review

- No space constraints or color figure charges

- Immediate publication on acceptance

- Inclusion in PubMed, CAS, Scopus and Google Scholar

- Research which is freely available for redistribution 